\title{
Takotsubo Syndrome as a Complication of Mild COVID-19: A Case Report
}

Jéssica Mayara Oséas, ${ }^{1,2}$ William Santos de Oliveira, ${ }^{\circledR}$ Bruna Aparecida Oliveira, ${ }^{1,2}{ }^{\circledR}$ Jayson Marcus Miranda, ${ }^{2}$ Raissa Gabriela Vieira Barros, ${ }^{2}{ }^{\circledR}$ Nestor Rodrigues de Oliveira Neto ${ }^{1}$

Department of Cardiology, Hospital Universitário Onofre Lopes, UFRN Medical School, ${ }^{1}$ Natal, RN - Brazil

Department of Cardiology, Hospital Casa de Saúde São Lucas, ${ }^{2}$ Natal, RN - Brazil

\section{Introduction}

As the number of cases of coronavirus disease 2019 (COVID-19) has reached tens of millions worldwide, the understanding of its clinical manifestations has grown. These have been described as a range of systemic repercussions resulting from an interplay between direct organ damage and exaggerated host immune response..$^{1,2}$ In a descriptive case series study of COVID-19 patients in Wuhan, ${ }^{2} 44 \%$ of patients had either elevated cardiac biomarkers or new changes in electrocardiogram (ECG) and imaging exams, and $34 \%$ of all cases had acute heart failure. Systemic diseases with increased adrenergic response may lead to stress cardiomyopathy, or Takotsubo syndrome, which is a rare condition with challenging diagnosis in the course of COVID-19. Herein we present a case of an elderly woman with mild COVID-19 who presented classical features of takotsubo syndrome on the 5 th day of illness.

\section{Case presentation}

A 90-year-old woman presented to the emergency unit with epigastric pain, nausea, and dyspnea for about 24 hours. She also complained of fever, cough, and coryza during the last five days. The patient had controlled hypertension with use of Losartan and denied other comorbidities. Her physical examination was unremarkable. She had an axillary temperature of 36.5 ${ }^{\circ} \mathrm{C}\left(97.7^{\circ} \mathrm{F}\right)$, heart rate of 92 beats per minute, respiratory rate of 18 breaths per minute, oxygen saturation of $96 \%$

\section{Keywords}

Takotsubo Cardiomyopathy, Stress Cardiomyopathy, Coronavirus Infections, Left ventricular dysfunction. while breathing ambient air, and blood pressure of 170/100 mm Hg.

A 12-lead ECG (Figure 1) showed sinus rhythm with widespread deep $\mathrm{T}$ wave inversion and very prolonged QTc interval. Immediate measurement of high-sensitivity cardiac troponin I yielded $522 \mathrm{ng} / \mathrm{L}$ (99th percentile, $9 \mathrm{ng} / \mathrm{L})$, followed by values of $636 \mathrm{ng} / \mathrm{L}$ and $756 \mathrm{ng} / \mathrm{L}$ three hours and six hours later, respectively. Also, a routine laboratory evaluation for patients with suspected COVID-19 showed mild leukopenia with lymphopenia, thrombocytopenia, elevated lactate dehydrogenase (LDH), and D-dimer of $800 \mathrm{ng} / \mathrm{mL}$ (reference range, $<500 \mathrm{ng} / \mathrm{mL}$ ). Chest computed tomography imaging was normal. The diagnosis of severe acute respiratory syndrome coronavirus 2 (SARS-CoV-2) was confirmed by a positive real-time reverse transcriptase polymerase chain reaction (RT-PCR).

Standard therapy for acute coronary syndrome (ACS) was started with aspirin, clopidogrel, enoxaparin, atorvastatin, and metoprolol, and the patient was admitted to the intensive care unit. Transthoracic echocardiogram revealed diffuse left ventricular (LV) akinesis in the middle chamber and apical segments, and preserved contractility of basal segments (Figure 2A). LV ejection fraction was $48 \%$, and the right ventricle had no signs of dysfunction or wall motion abnormalities. Cardiac catheterization was performed thereafter and showed no significant coronary lesions. Ventriculography confirmed the presence of apical hypokinesis consistent with takotsubo syndrome (Figure 2B).

The patient had rapid improvement of symptoms during hospitalization. Five days after admission, highsensitivity troponin I value was $58.8 \mathrm{ng} / \mathrm{L}$, and ECG and echocardiographic findings were normalized. The possible recurrence of takotsubo syndrome and the lack

Mailing Address: Nestor Rodrigues de Oliveira Neto

Hospital Universitário Onofre Lopes

Av. Nilo Peçanha, 620. Postal Code: 59012-300, Petrópolis, Natal, RN - Brazil.

E-mail: superintendencia.huol@ebserh.gov.br 


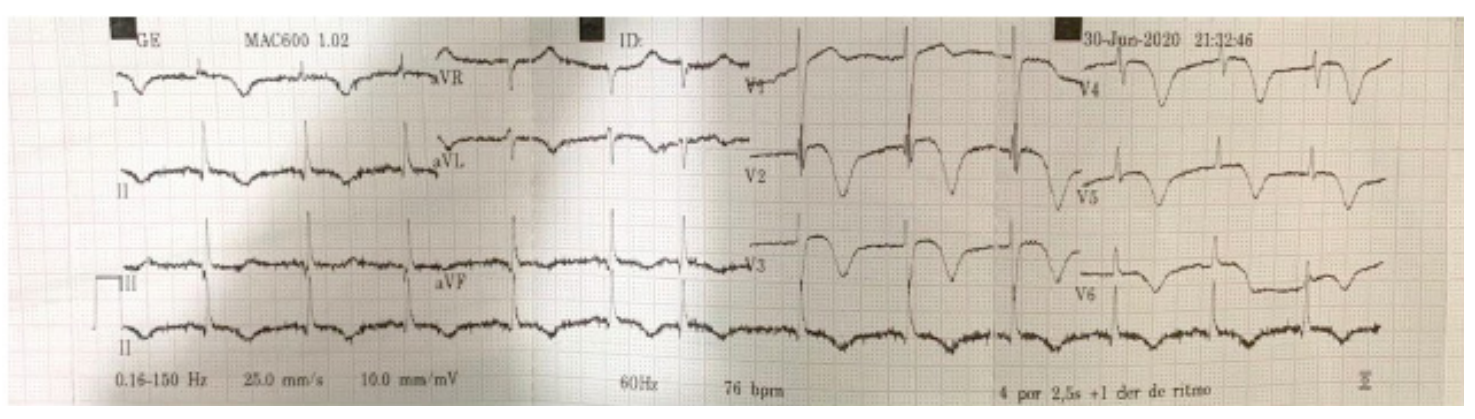

Figure 1 - Initial 12-lead ECG showing sinus rhythm and widespread, deep T wave inversion with a prolonged QTc interval.

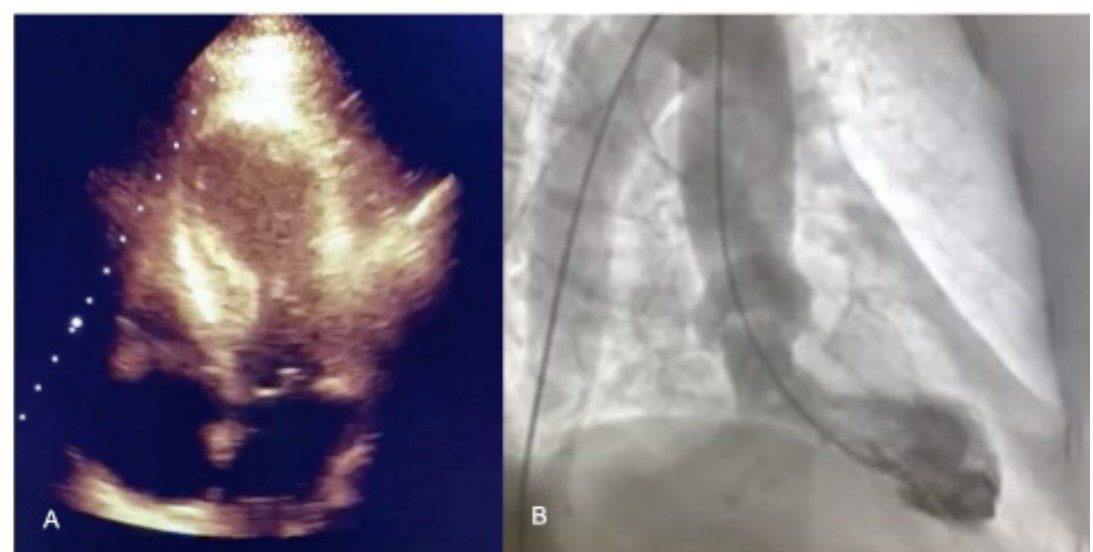

Figure 2 - Transthoracic echocardiogram (A) and ventriculography (B) images show the presence of extensive apical hypokinesia with preserved motility in the basal segments, characterizing the apical ballooning pattern observed in patients with takotsubo syndrome.

of a standardized long-term therapy were discussed with the patient, and she was discharged for clinical and cardiac imaging follow-up.

\section{Discussion}

COVID-19 has been associated with a wide range of cardiovascular manifestations that include arrhythmias, heart failure, acute ischemia, myocarditis, and venous thromboembolism. ${ }^{1}$ In a case series ${ }^{3}$ of COVID-19 patients in China, those with elevated cardiac biomarkers were shown to have a greater need for mechanical ventilation $(22.0 \%$ vs $4.2 \%, \mathrm{p}<.001)$ and higher mortality $(51.2 \%$ vs $4.5 \%, \mathrm{p}<.001)$. Cardiac injury was an independent predictor of mortality both during the time from symptom onset (hazard ratio, 4.26 [95\% CI, 1.929.49]) and from admission to end point, which was reproduced in other cohorts. ${ }^{1}$ Although it is known that heart involvement is associated with worse prognosis in COVID-19, the understanding of the mechanisms of cardiac injury is hindered by the scarcity of imaging and histologic data available. It is currently suggested that the cytokine storm and the ensuing inflammatory stress present in patients with severe COVID-19 play a major role in both new myocardial damage and decompensation of pre-existing heart disease. ${ }^{1-3}$ The milieu of hypoxic respiratory failure and circulatory compromise of the critically ill promotes further myocardial insult. Additionally, individual reports depicting ECG and cardiovascular magnetic resonance (CMR) features characteristic of acute myocarditis imply that a direct infection of the heart muscle occurs in a subset of patients, which is further supported by cases with histologic evidence of myocardial inflammation and the detection of viral genome in heart tissue samples. ${ }^{1,4}$ Despite the frequency of heart involvement in COVID-19, 
takotsubo syndrome has been rarely reported in this condition. Takotsubo syndrome consists of a transient myocardial dysfunction putatively induced by intense adrenergic stimulation, either by physical or emotional stress..$^{5}$ Also, the misdiagnosis with ACS still hinders estimating the true incidence of takotsubo syndrome. It is expected that $1-3 \%$ of individuals hospitalized for suspected myocardial infarction will be diagnosed with the syndrome. As in our case, takotsubo syndrome occurs mostly in postmenopausal women, with a female to male ratio of 9:1 and a mean age of 66 years. ${ }^{5}$ Although different diagnostic criteria have been proposed, the presence of reversible LV contraction abnormalities that extend beyond vascular territories is considered the primary manifestation of takotsubo syndrome. Apical ballooning, which occurred in our patient, is typical, but not always present. Likewise, deep and widespread $\mathrm{T}$ wave inversion with QTc prolongation is a specific ECG finding that may help differentiating takotsubo syndrome from myocardial infarction. ${ }^{5}$

The mechanisms by which takotsubo syndrome may occur in the course of coronavirus infection are uncertain, although the inflammatory state and increased adrenergic drive are plausible inciting factors. ${ }^{6}$ Moreover, an association of takotsubo syndrome with infectious diseases, leading to acute critical illness, has been previously recognized. ${ }^{1,6}$ The course of takotsubo syndrome in patients with COVID-19 seems no to differ from the classical presentation, and in previously published cases $^{6}$ its diagnosis relied on the presence of acute ventricular dysfunction with apical ballooning. Nevertheless, while in previous reports, ${ }^{6}$ those patients who developed typical features of takotsubo syndrome also had severe respiratory compromise with need for supplemental oxygen and sometimes mechanical ventilation, our patient had mild symptoms of COVID-19 and unremarkable chest CT. The occurrence of takotsubo syndrome in patients with mild viral diseases is documented, ${ }^{5}$ although such reports are rare. Additionally, as happened in other reports involving patients with COVID-19, CMR and endomyocardial biopsy have been deferred to avoid futile medical investigations in patients on transmissionbased precautions. Further evaluation could have been valuable for ruling out the presence of acute myocarditis as a mimic of takotsubo syndrome. Finally, subsequent outpatient follow-up data will help to understand the long-term course of takotsubo syndrome, and other forms of cardiac disease in patients with COVID-19.

\section{Conclusions}

Takotsubo syndrome is a rare event in patients with COVID-19. Its mechanisms and relationship to other forms of cardiac injury caused by SARS-CoV-2 are still uncertain. Nevertheless, awareness of the condition even in patients with mild disease is important to guide appropriate management and to further understand the array of systemic and cardiovascular implications of COVID-19.

\section{Potential Conflict of Interest}

No potential conflict of interest relevant to this article was reported.

\section{Sources of Funding}

There were no external funding sources for this study.

\section{Study Association}

This study is not associated with any thesis or dissertation work.

\section{Author contributions}

Conception and design of the research: Oséas JM, de Oliveira WS, de Oliveira Neto NR. Acquisition of data: Oséas JM, Oliveira BA, Barros RGV. Analysis and interpretation of the data: Oséas JM, de Oliveira WS, Miranda MR, de Oliveira Neto NR. Writing of the manuscript: Oséas JM, de Oliveira WS, de Oliveira Neto NR. Critical revision of the manuscript for intellectual content: Oséas JM, de Oliveira WS, Oliveira BA, Barros RGV, de Oliveira Neto NR.

\section{Ethics approval and consent to participate}

This article does not contain any studies with human participants or animals performed by any of the authors. 


\section{References}

1. Nishiga M, Wang DW, Han Y, Lewis DB, Wu JC. COVID-19 and cardiovascular disease: from basic mechanisms to clinical perspectives. Nat Rev Cardiol. 2020;17(9):543-58. doi: 10.1038/s41569-020-0413-9.

2. Chen T, Wu D, Chen H, Yan W, Yang D, Chen G, et al. Clinical characteristics of 113 deceased patients with coronavirus disease 2019: retrospective study. BMJ. 2020;368:m1091. doi: 10.1136/bmj.m1091.

3. Shi S, Qin M, Shen B, Cai Y, Liu T, Yang F, et al. Association of Cardiac Injury With Mortality in Hospitalized Patients With COVID-19 in Wuhan, China. JAMA Cardiol. 2020;5(7):802-10. doi: 10.1001/ jamacardio.2020.0950.
4. Lindner D, Fitzek A, Bräuninger H, Aleshcheva G, Edler C, Meissner $\mathrm{K}$, et al. Association of cardiac infection with SARS-CoV-2 in confirmed COVID-19 autopsy cases. JAMA Cardiol. 2020;5(11):1281-85. doi: 10.1001/ jamacardio.2020.3551.

5. Chazal HM, Del Buono MG, Keyser-Marcus L, Ma L, Moeller FG, Berrocal D, et al. Stress cardiomyopathy diagnosis and treatment: JACC stateof-the-art review. J Am Coll Cardiol. 2018;72(16):1955-71. doi: 10.1016/j. jacc.2018.07.072.

6. Singh S, Desai R, Gandhi Z, Fong HK, Doreswamy S, Desai V, et al. Takotsubo syndrome in patients with COVID-19: a systematic review of published cases. SN Compr Clin Med. 2020:1-7. doi: 10.1007/s42399020-00557-w. 